\title{
The Evaluation of Myocardial Performance Index in Patients with COVID-19: An Echocardiographic Follow-up Study"
}

\author{
Hakan Kaya ${ }^{1}$, Ramazan Asoğlu ${ }^{2}$, Abdulmecit Afşin ${ }^{3}$, hakan tibilli ${ }^{1}$, Ercan Kurt ${ }^{1}$, Safiye \\ Kafadar $^{1}$, Umut Gülaçtı ${ }^{1}$, and Hüseyin Kafadar ${ }^{1}$ \\ ${ }^{1}$ Adiyaman University Faculty of Medicine \\ ${ }^{2}$ Adiyaman Universitesi \\ ${ }^{3}$ Adiyaman University
}

October 29, 2020

\begin{abstract}
INTRODUCTION: The epidemic of pneumonia caused by a new coronavirus disease 2019 (COVID-19) rapidly spread all over the world. Pathophisyology of the cardiovascular effects of COVID-19 is still not well known. Myocardial dysfunction may occur in cytokine-based immune reactions. Myocardial performance index (MPI) is a feasible parameter that reflects systolic and diastolic cardiac functions. We aimed to evaluate the MPI in patients with COVID-19. METHODS: The study consisted of 40 patients diagnosed with COVID-19 who had mild pneumonia and did not need intensive care treatment. All patients underwent echocardiographic evaluation. The MPI and laboratory parameters were compared between the acute period of infection and after clinical recovery in patients with COVID-19. RESULTS: Statistically significant higher MPI $(0.56 \pm 0.09$ versus $0.44 \pm 0.07$, $\mathrm{p}<0.001)$, longer isovolumic relaxation time $(112.3 \pm 13.4$ versus $91.8 \pm 12.1 \mathrm{~ms}, \mathrm{p}<0.001)$, longer deceleration time $(182.1 \pm$ 30.6 versus $161.5 \pm 43.5 \mathrm{~ms}, \mathrm{p}=0.003)$, shorter ejection time $(279.6 \pm 20.3$ versus $298.8 \pm 36.8 \mathrm{~ms}, \mathrm{p}<0.001)$ and higher E/A ratio ( $1.53 \pm 0.7$ versus $1.22 \pm 0.4, \mathrm{p}<0.001$ ), were observed during acute period of infection compared to ones after clinical recovery. Compared with basal values, no significant change in left ventricular (LV) systolic ejection fraction was observed after clinical recovery $(60.3 \pm 3.2 \%$ versus $61.7 \pm 2.4 \%, \mathrm{p}>0.05)$. CONCLUSION: Our study showed that although, LV systolic function appear normal in COVID-19 patients, they have globally reversible LV diastolic dysfunction, based on tissue Doppler derived MPI. This could be due to isolated subclinical diastolic dysfunction. The underlying mechanism and its clinical significance can be established by further studies.
\end{abstract}

The Evaluation of Myocardial Performance Index in Patients with COVID-19: An Echocardiographic Follow-up Study

\section{ABSTARCT}

INTRODUCTION The epidemic of pneumonia caused by a new coronavirus disease 2019 (COVID-19) rapidly spread all over the world. Pathophisyology of the cardiovascular effects of COVID-19 is still not well known. Myocardial dysfunction may occur in cytokine-based immune reactions. Myocardial performance index (MPI) is a feasible parameter that reflects systolic and diastolic cardiac functions. We aimed to evaluate the MPI in patients with COVID-19.

METHODS The study consisted of 40 patients diagnosed with COVID-19 who had mild pneumonia and did not need intensive care treatment. All patients underwent echocardiographic evaluation. The MPI and laboratory parameters were compared between the acute period of infection and after clinical recovery in patients with COVID-19.

RESULTS Statistically significant higher MPI (0.56 \pm 0.09 versus $0.44 \pm 0.07, \mathrm{p}<0.001)$, longer isovolumic 
relaxation time $(112.3 \pm 13.4$ versus $91.8 \pm 12.1 \mathrm{~ms}, \mathrm{p}<0.001)$, longer deceleration time $(182.1 \pm 30.6$ versus $161.5 \pm 43.5 \mathrm{~ms}, \mathrm{p}=0.003)$, shorter ejection time $(279.6 \pm 20.3$ versus $298.8 \pm 36.8 \mathrm{~ms}, \mathrm{p}<0.001)$ and higher $\mathrm{E} / \mathrm{A}$ ratio (1.53 \pm 0.7 versus $1.22 \pm 0.4, \mathrm{p}<0.001)$, were observed during acute period of infection compared to ones after clinical recovery. Compared with basal values, no significant change in left ventricular (LV) systolic ejection fraction was observed after clinical recovery $(60.3 \pm 3.2 \%$ versus $61.7 \pm 2.4 \%, \mathrm{p}>0.05)$.

CONCLUSION Our study showed that although, LV systolic function appear normal in COVID-19 patients, they have globally reversible LV diastolic dysfunction, based on tissue Doppler derived MPI. This could be due to isolated subclinical diastolic dysfunction. The underlying mechanism and its clinical significance can be established by further studies.

Key words: COVID-19, echocardiography, myocardial performance index.

\section{INTRODUCTION}

Wuhan, China became the center of an epidemic of unknown pneumonia on December, 3, 2019 (1-4). Blood samples, oral swabs, bronchoalveolar lavage fluid, and anal swabs collected from patients with severe pneumonia were analyzed. This novel pathogen, enveloped RNA beta-coronavirus, was named severe acute respiratory syndrome coronavirus 2 (5). Subsequently, the disease caused by this virus was called coronavirus disease 2019 (COVID-19). COVID-19 has become a life-threatening public health emergency internationally (6). COVID-19 typically presents with respiratory tract symptoms including fever, dry cough, and dyspnea. The disease can progress to pneumonia and acute respiratory distress syndrome (7). The physiopathology of COVID-19 infection is not clear. However, an extreme immune response and cytokine storm are thought to have a pivotal roles in the etiopathogenesis of the disease. Our knowledge of the cardiovascular effects of COVID-19 is limited. Studies have reported that the left and right ventricular ejection fractions may be reduced by a cytokine-mediated systemic response to infections by increasing the end-diastolic and end-systolic volumes of both ventricles (8). However, it is not known whether systolic and diastolic dysfunction of the left ventricle (LV) develops in patients with COVID-19.

Two-dimensional and Doppler echocardiography provide important information about the diastolic and systolic function of the LV. The myocardial performance index (MPI) is a reliable echocardiographic index that combines diastolic and systolic measurements (9). We evaluated the MPI of patients with COVID-19.

\section{METHODS}

\section{Study Population}

The present study consisted of fourty healthy control subjects and 40 patients diagnosed with COVID-19 who had mild pneumonia and did not need intensive care treatment. Patients with atrioventricular conduction disturbance, non-sinus rhythm on ECG, ventricular extra-systoles, valvular heart disease, and a history of chronic heart failure, chronic rheumatic heart disease, atrial dysrhythmia, a permanent cardiac pacemaker, or acute coronary syndrome were excluded from the study. The clinical history and laboratory data of patients and results of reverse transcription-polymerase chain reaction (RT-PCR) of oropharyngeal swabs were evaluated. All patients had a positive RT-PCR result. All patients with COVID-19 were treated with vitamin $\mathrm{C}$, hydroxychloroquine, and/or favipravir. All participants were recruited from Adiyaman University Hospital. This study was conducted prospectively according to the principles of the Declaration of Helsinki. The study protocol was approved by the institutional medical ethics committee (2020/5-25). Written informed consent was obtained from every participant.

\section{Echocardiographic Examination}

Transthoracic echocardiographic examination was performed in all subjects using a Vivid 5S (GE Healthcare Systems, Horten, Norway) with a $2.0-3.5-\mathrm{MHz}$ transducer. Transthoracic echocardiographic examination was performed at the acute stage of infection and after clinical recovery in patients with COVID-19. The recovery echocardiography exam was performed on patients who were still hospitalised but had recovered symptomatically and according to laboratory findings. The average time interval between the baseline and 
recovery echocardiography exam was about $28 \pm 3,4$ days. Doppler and Two-dimensional echocardiography were done in the standard views according to the guidelines of the American Society of Echocardiography (10). The modified Simpson's method was used to calculate the left ventricular ejection fraction (LVEF) (11). We used apical four-chamber views by putting the sample volume between the tips of the mitral leaflets for the pulsed-wave Doppler recordings of the mitral inflow velocities. The peak late (A) and early (E) transmitral filling velocities and the ratio of transmitral early to late peak velocities (E/A) were measured as conventional Doppler indices. The time between closing the aortic valve and opening the mitral valve was defined as the isovolumic relaxation time (IRT). The time between closing the mitral valve and opening the aortic valve was accepted as the isovolumic contraction time (ICT). Ejection time (ET) was defined as the time between opening and closing of the aortic valve on the LV out flow velocity profile. Pulsed wave tissue Doppler imaging was performed from an apical four-chamber view with a sample volume of $2 \mathrm{~mm}$ placed on the medial wall of the mitral annulus. High frequency signals were excluded by making filter settings. The scanning intervals of Doppler ultrasound were obtained from the mitral annular velocity intervals. MPI was calculated by using the equation: MPI $=(\mathrm{ICT}+\mathrm{IRT}) / \mathrm{ET}(12)$. Tissue Doppler measurements were obtained from an average of five consecutive cardiac cycles. The calculation of tissue Doppler-derived MPI is presented in Figure 1.

\section{Laboratory Parameters}

Blood samples were studied on days $0,7,14$, and 28. An immunofluorescence assay was used to obtain COVID-19 antibody titers. Respiratory secretions were sent for RT-PCR testing. Cardiac troponin I (cTnI) was studied with Immulate (Bio, DPC) from blood samples. The lactate dehydrogenase (LDH), creatinine kinase (CK), and creatinine levels were analyzed using an Architect c8000 Chemistry System (Abbott Diagnostics, Abbott Park, IL, USA) with commercial kits (Abbott Diagnostics). Hemoglobin and complete white blood counts (WBC), including neutrophil and lymphocyte counts, were measured using an automated CELL-DYN Ruby hematology analyzer (Abbott Diagnostics) and expressed as ×103 cells/mm3

\section{Statistical analysis}

SPSS v. 22.0 (Chicago, USA) was used for the analyses. Categorical variables are presented as numbers and percentages, and were compared with the Chi-square test. Continuous variables were tested for distribution normality with the Kolmogorov-Smirnov test. Continuous variables are presented as the mean \pm standard deviation. An independent t-test or Mann-Whitney U-Test was used to compare the variables between the two groups, as appropriate. The acute period of infection and clinical recovery values were compared using the paired t-test if the data were normally distributed. A P-value below 0.05 was considered statistically significant.

\section{RESULTS}

This study enrolled 40 consecutive patients (26 males (65\%); mean age, $54 \pm 11$ years) and 40 healthy control subjects (24 males $(60 \%)$; mean age, $52 \pm 13$ years). The patients were admitted to hospital with a fever $>38^{\circ} \mathrm{C}$, cough, sore throat, rhinorrhea, shortness of breath, headache, myalgia, diarrhea. The PCR test was positive in all patients. The mean interval between the first echocardiogram and symptom onset was $9.3 \pm 5.5$ days. The clinical characteristics including; age, gender, smoking, hypertension, diabetes, systolic blood pressure, diastolic blood pressure, heart rate were similar between the patients and healthy control subjects ( $>0.05$, for all). ). Compared with the healthy controls, patients with COVID-19 had a significant higher MPI $(0.56 \pm 0.09$ versus $0.41 \pm 0.06, \mathrm{p}<0.001)$, longer IRT $(112.3 \pm 13.4$ versus $90.6 \pm 11.2 \mathrm{~ms}, \mathrm{p}<0.001)$, longer deceleration time $(182.1 \pm 30.6$ versus $160.8 \pm 42.7 \mathrm{~ms}, \mathrm{p}=0.003)$, shorter ET $(279.6 \pm 20.3$ versus $299.6 \pm 34.7 \mathrm{~ms}$, $\mathrm{p}<0.001)$ and higher $\mathrm{E} / \mathrm{A}$ ratio $(1.53 \pm 0.7$ versus $1.21 \pm 0.3, \mathrm{p}<0.001)$. LVEF was similar in the patients with COVID-19 and healthy controls $(60.3 \pm 3.2 \%$ versus $61.9 \pm 4.8 \%, \mathrm{p}>0.05)$. ICT was similar in both groups $(44.3 \pm 7.8$ versus $41.8 \pm 8.7 \mathrm{~ms}, \mathrm{p}>0.05)$. Compared with the healthy controls, the patients with COVID-19 had a significantly higher cTnI $(0.96 \pm 0.12$ versus $0.10 \pm 0.01 \mathrm{ng} / \mathrm{ml}, \mathrm{p}<0.001), \mathrm{LDH}(486.8 \pm 118.6$ versus $316.4 \pm 108.9 \mathrm{IU} / \mathrm{L}, \mathrm{p}=0.002) \mathrm{WBC}\left(12.3 \pm 4.7\right.$ versus $\left.7.0 \pm 2.610^{3} / \mu \mathrm{l}, \mathrm{p}<0.001\right)$, neutrophil count $(9.7 \pm 6.2$ versus $\left.4.2 \pm 1.510^{3} / \mu \mathrm{l}, \mathrm{p}<0.001\right)$ and lower lymphocyte count $\left(0.9 \pm 0.2\right.$ versus $\left.2.8 \pm 0.610^{3} / \mu \mathrm{l}, \mathrm{p}<0.001\right)$. The 
creatinine, CK and hemoglobin levels were similar in both groups ( $\mathrm{p}>0.05$, for all). Table 1 summarizes the clinical characteristics and laboratory and echocardiographic findings of the study groups.

The systolic and diastolic blood pressure and heart rate were similar between the acute period of infection and after clinical recovery ( $>00.05$, for all). Compared with the acute period of infection in patients with COVID-19, after clinical recovery there was a significantly lower MPI $(0.44 \pm 0.07$ versus $0.56 \pm 0.09$, $\mathrm{p}<0.001)$, shorter IRT $(91.8 \pm 12.1$ versus $112.3 \pm 13.4 \mathrm{~ms}, \mathrm{p}<0.001)$, shorter deceleration time $(161.5 \pm 43.5$ versus $182.1 \pm 30.6 \mathrm{~ms}, \mathrm{p}=0.003)$, longer $\mathrm{ET}(298.8 \pm 36.8$ versus $279.6 \pm 20.3 \mathrm{~ms}, \mathrm{p}<0.001)$ and lower $\mathrm{E} / \mathrm{A}$ ratio $(1.22 \pm 0.6$ versus $1.53 \pm 0.7, \mathrm{p}<0.001)$. $)$. LVEF was similar in the acute period of infection and after clinical recovery $(60.3 \pm 3.2 \%$ versus $60.7 \pm 3.8 \%, \mathrm{p}>0.05)$. ICT was similar in the acute period of infection and after clinical recovery $(44.3 \pm 7.8$ versus $40.6 \pm 9.7 \mathrm{~ms}, \mathrm{p}>0.05)$. Compared with the acute period of infection in patients with COVID-19, after clinical recovery there was a significantly lower cTnI $(0.11 \pm 0.08$ versus $0.96 \pm 0.12$ $\mathrm{ng} / \mathrm{ml}, \mathrm{p}<0.001), \mathrm{LDH}(334.5 \pm 110.9$ versus $486.8 \pm 118.6 \mathrm{IU} / \mathrm{L}, \mathrm{p}=0.004)$, WBC $(7.2 \pm 3.1$ versus $12.3 \pm 4.7$ $103 / \mu \mathrm{l}, \mathrm{p}<0.001)$, neutrophil count $\left(4.5 \pm 1.8\right.$ versus $\left.9.7 \pm 6.210^{3} / \mu \mathrm{l}, \mathrm{p}<0.001\right)$ and higher lymphocyte count $\left(2.1 \pm 0.4\right.$ versus $\left.0.9 \pm 0.210^{3} / \mu \mathrm{l}, \mathrm{p}<0.001\right)$. The creatinine, CK and hemoglobin levels were similar between the acute period of infection and after clinical recovery ( $\mathrm{p}>0.05$, for all). Table 2 gives the clinical characteristics, laboratory and echocardiographic findings of the patients with COVID-19 in the acute period of infection and after clinical recovery.

\section{DISCUSSION}

This is the first echocardiographic follow-up study to evaluate the systolic and diastolic function of the left ventricle in COVID-19 patients. Our data showed that LV performance was impaired subclinically during the acute period of COVID-19 and this impaired performance recovered with clinical improvement.

Since the first case was reported at the end of 2019, the COVID-19 outbreak has become a pandemic. The high transmission ratio of the virus has made it a threat to public health globally $(13,14)$. Coronaviruses are non-segmented positive-sense RNA viruses that are broadly distributed in humans and other mammals, such as camels, bats, mice, dogs, and cats. (15). Coronaviruses can cause respiratory, enteric, cardiovascular, and neurological diseases (16-19). The most common symptoms of COVID-19 are fever (in $88.7 \%$ of patients) and a dry cough (in $67.8 \%$ of patients), followed by headache, fatigue, or shortness of breath. The overall mortality rate is $2.3 \%$ and those with pre-existing comorbid conditions have higher case mortality rates: diabetes $7.3 \%$, chronic respiratory disease $6.3 \%$, hypertension $6.0 \%$ cardiovascular disease $10.5 \%$, and cancer $5.6 \%(20)$.

We assessed the left ventricular diastolic and systolic function using several echocardiographic parameters. Although systolic and diastolic dysfunction appear together, there are few Doppler echocardiogram variables that combine systolic and diastolic measurements. We used the MPI, which includes the systolic contraction and diastolic relaxation periods of the left ventricle (21). MPI is not significantly affected by pre-load, afterload, sample volume location, age, or rhythm $(22,23)$. Therefore, it is a reliable index. The value of MPI in left ventricular dysfunction has been confirmed in patients with symptomatic heart failure with nonischemic or ischemic etiology and idiopathic dilated cardiomyopathy $(21,22)$. In the present study, MPI was higher in the acute disease period in the patients with COVID-19, but regressed significantly after clinical recovery.

The pathophysiological and cardiovascular effects of COVID-19 are not clear. However, the disease is thought to be associated with an excessive immune response triggered by the virus. Studies have found that COVID19 patients have high levels of interleukin (IL)-1 beta, IL-6, interferon gamma, and monocyte chemoattractant protein-1, which probably led to an activated T-helper-1 cell response (7). Studies of coronavirus and influenza outbreaks suggest that viral infections can trigger a systemic inflammatory response that causes acute coronary syndromes, arrhythmias, and heart failure (23-26). Similarly, underlying cardiovascular diseases may be exacerbated or new cardiac pathologies may be induced during COVID-19. Acute coronary events may be due to increased myocardial demand triggered by infections that accelerate myocardial damage or infarction. Alternatively, circulating cytokines released during severe systemic inflammatory stress may cause 
instability and rupture of atherosclerotic plaque. Patients with heart failure are also prone to hemodynamic decompensation during the stress of severe infections. During most influenza outbreaks, more patients die of cardiovascular causes than from pneumonia (27). COVID-19 causes myocardial inflammation and myocarditis (28-30). In our patients, the elevated cTnI might reflect the myocardial damage. cTnI is part of the contractile apparatus in cardiomyocytes and is expressed only in the heart (31). As a result of its organ specificity, cTnI can be used to diagnose cardiomyocyte damage (32). The elevated LDH might reflect the tissue damage and inflammation (33). We assumed that the temporary impairment in echocardiographic parameters in the acute phase was caused by proinflammatory mediators, such as tumor necrosis factor (TNF) and the IL- 1 and -6 families of cytokines, which have significant negative inotropic effects (34). Myocardial function may also be impaired with direct COVID-19 myocyte infection, leading to active myocarditis. Reported data on the severity, extent, and short- and long-term cardiovascular effects of COVID-19 are limited. Our study has limitations. The main study limitation is the small sample size. Another limitation is that the circulatory levels of cytokines such as IL-1, IL-6, and TNF could not be measured. Further studies that measure the circulatory levels of these cytokines and echocardiographic variables are needed based on the current hypothesis of cytokine-mediated temporary impairment of left ventricular performance.

In conclusion, our study showed that although, LV systolic function appear normal in COVID-19 patients, they have globally reversible LV diastolic dysfunction, based on tissue Doppler derived MPI. This could be due to isolated subclinical diastolic dysfunction. The underlying mechanism and its clinical significance can be established by further studies.

Conflict interests: The authors declare that they have no competing interests.

Funding : This study received no grant funding from any agency in the public, commercial or not-for-profit sectors.

Acknowledgements : Our article has been accepted as a e-poster presentation in EACVI - Best of Imaging 2020 (Number: 90512).

Availability of data and materials: The datasets used and/or analyzed during the current study are available from the corresponding author on reasonable request.

Author contributions: Concept/design: HK, UG, HT, EK, Data analysis/interpretation: HK, HT, EK, Drafting article: AA, RA, EK, SK, Critical revision of article: AA, RA, Approval of article: HK, RA, HT, AA, UG, EK, SK, HK, Statistics: HK, UG, Funding: HK, EK Data collection: HK.

\section{REFERENCES}

1. Wang C, Horby PW, Hayden FG, et al. A novel coronavirus outbreak of global health concern. Lancet 2020; 395: 470-3.

2. Hsu LY, Chia PY, Vasoo S. A midpoint perspective on the COVID-19 pandemic. Singapore Med J 2020 ;

3. Lum LHW, Tambyah PA. Outbreak of COVID-19 - an urgent need for good science to silence our fears? Singapore Med J 2020;61(2):55-7

4. Kok SXS, Shah MT, Cheong WK, et al. Dealing with COVID-19: initial perspective of a small radiology department. Singapore Med J 2020;

5. Lu R, Zhao X, Li J, et al. Genomic characterisation and epidemiology of 2019 novel coronavirus: implications for virus origins and receptor binding. Lancet 2020; 395: 565-74.

6. Ksiazek TG, Erdman D, Goldsmith CS, et al. A novel coronavirus associated with severe acute respiratory syndrome. N Engl J Med 2003; 348: 1953-66.

7. Huang C,Wang Y, Li X, et al. Clinical features of patients infected with 2019 novel coronavirus in Wuhan, China. Lancet 2020; 395: 497-506.

8. Parrillo JE. Pathogenetic mechanism of septic shock. N Engl J Med 1993; 328:1471-77.

9. Tei C: New non-invasive index for combined systolic and diastolic ventricular function. J Cardiol 1995; 26: $135-6$. 
10. Nagueh SF, Smiseth OA, Appleton CP, et al. Recommendations for the Evaluation of Left Ventricular Diastolic Function by Echocardiography: An Update from the American Society of Echocardiography and the European Association of Cardiovascular Imaging. J Am Soc Echocardiogr. 2016;29(4):277-314.

11. Lang RM, Badano LP, Mor-Avi V, et al. Recommendations for Cardiac Chamber Quantification by Echocardiography in Adults: An Update from the American Society of Echocardiography and the European Association of Cardiovascular Imaging. J Am Soc Echocardiogr. 2015; 28: 1-39.

12. Tei C, Nishimura RA, Seward JB, et al. Noninvasive Doppler derived myocardial performance index: correlation with simultaneous measurements of cardiac catheterization measurements. J Am Soc Echocardiogr 1997; 10: 169-78.

13. World Health Organization. Pneumonia of unknown cause - China. Accessed January 5, 2020.https://www.who.int/csr/don/05-january-2020-pneumonia-of-unkown-cause-china/en/

14. World Health Organization. Novel coronavirus - China. Accessed January 12, 2020. https://www.who.int/csr/don/12-january-2020- novel-coronavirus-china/en/

15. Su S, Wong G, Shi W, et al. Epidemiology, genetic recombination, and pathogenesis of coronaviruses. Trends Microbiol 2016; 24: 490-502.

16. Yin Y, Wunderink RG. MERS, SARS and other coronaviruses as causes of pneumonia. Respirology 2018; 23: 130-37.

17. Drosten C, Günther S, Preiser W, et al. Identification of a novel coronavirus in patients with severe acute respiratory syndrome. N Engl J Med 2003; 348: 1967-76.

18. Zaki AM, van Boheemen S, Bestebroer TM, et al. Isolation of a novel coronavirus from a man with pneumonia in Saudi Arabia. N Engl J Med 2012; 367: 1814-20.

19. Weiss SR, Leibowitz JL. Coronavirus pathogenesis. Advances in virus research 2011; 81: 85-164.

20. Wu Z, McGoogan JM. Characteristics of and Important Lessons From the Coronavirus Disease 2019 Outbreak in China: Summary of a Report of 72314 Cases From the Chinese Center for Disease Control and Prevention. JAMA 2020; 323: 1239-42.

21. Dujardin KS, Tei C, Yeo TC, et al. Prognostic value of a Doppler index combining systolic and diastolic performance in idiopathic-dilated cardiomyopathy. Am J Cardiol 1998; 82: 1071-76.

22. Harjai KJ, Scott L, Vivekananthan K, et al. The Tei index: a new prognostic index for patients with symptomatic heart failure. J Am Soc Echocardiogr 2002; 15: 864-8.

23. Madjid M, Miller CC, Zarubaev VV, et al. Influenza epidemics and acute respiratory disease activity are associated with a surge in autopsy-confirmed coronary heart disease death: results from 8 years of autopsies in 34,892 subjects. Eur Heart J 2007; 28: 1205-10.

24. Kwong JC, Schwartz KL, Campitelli MA, et al. Acute myocardial infarction after laboratory-confirmed influenza infection. N Engl J Med 2018; 378: 345-53.

25. Madjid M, Connolly AT, Nabutovsky Y, et al. Effect of high influenza activity on risk of ventricular arrhythmias requiring therapy in patients with implantable cardiac defibrillators and cardiac resynchronization therapy defibrillators. Am J Cardiol 2019; 124: 44- 50.

26. Kytömaa S, Hegde S, Claggett B, et al. Association of influenza-like illness activity with hospitalizations for heart failure: the Atherosclerosis Risk in Communities Study. JAMA Cardiol 2019; 4: 363-9.

27. Madjid M, Casscells SW. Of birds and men: cardiologists' role in influenza pandemics. Lancet 2004; 364: 1309 .

28. Sala S, Peretto G, Gramegna M, et al. Acute myocarditis presenting as a reverse Tako-Tsubo syndrome in a patient with SARS-CoV-2 respiratory infection. Eur Heart J. 2020,41(19):1861-2.

29. Zhou R. Does SARS-CoV-2 cause viral myocarditis in COVID-19 patients? Eur Heart J 2020;41:2123.

30. Shi S, Qin M, Shen B, et.al. Association of Cardiac Injury With Mortality in Hospitalized Patients With COVID-19 in Wuhan, China. JAMA Cardiol. 2020 Mar 25 : e200950. https://doi.org/10.1001/jamacardio.2020.0950

31. Reichlin T, Hochholzer W, Bassetti S, et al. Early diagnosis of myocardial infarction with sensitive cardiac troponin assays. N Engl J Med 2009; 361: 858-67.

32. Keller T, Zeller T, Ojeda F, et al. Serial changes in highly sensitive troponin I assay and early diagnosis of myocardial infarction. JAMA 2011; 306: $2684-93$. 
33. Lee N, Hui D, Wu A, et al. A major outbreak of SARS in Hong Kong. N Engl J Med 2003; 348: 1986-94.

34. Mann DL. Inflammatory mediators and the failing heart: past, present and the foreseeable future. Circ Res 2002; 91:988-98.

Figure legend

Figure 1. Calculation of tissue Doppler derived left ventricular myocardial performance index. 